\title{
Etiology of hypercoagulable state in women with recurrent fetal loss without other causes of miscarriage from Southern Italy: new clinical target for antithrombotic therapy
}

\author{
Maristella D'Uva' \\ Pierpaolo Di Micco² \\ Ida Strina' \\ Antonio Ranieri' \\ Carlo Alviggi' \\ Antonio Mollo' \\ Francesca Fabozzi' \\ Lucia Cacciapuoti' \\ Maria Teresa Scotto di Frega' \\ Mariateresa lannuzzo ${ }^{2}$ \\ Giuseppe De Placido' \\ 'Dipartimento Universitario di \\ Scienze Ostetriche Ginecologiche \\ e Medicina della Riproduzione, \\ Area Funzionale di Medicina della \\ Riproduzione ed Endoscopia \\ Ginecologica, Università degli \\ Studi di Napoli Federico II, via \\ Pansini 5 Building 9, 80। 3 I, Naples, \\ Italy; ${ }^{2}$ Internal Medicine Division, \\ Fatebenefratelli Hospital of Naples, \\ Naples, Italy
}

Correspondence: Pierpaolo Di Micco Fatebenefratelli Hospital of Naples, Italy, Emergency Room Nad Internal Medicine, via Manzoni 220, Naples, Italy

ZIP code 80100

Tel $+393398078 I 46$

$\mathrm{Fax}+39815648290$

Email pdimicco@libero.it
Background: Recurrent fetal loss (RPL) is one of the most common cause of sterility. Several studies identified thrombophilia as the principal cause of recurrent pregnancy loss. However, reported studies often do not evaluate other causes of miscarriages in their inclusion and exclusion criteria. So the aim of our study was to investigate the role of inherited thrombophilia in patients with RPL and without other causes of RPL.

Patients and methods: Patients with 2 or more first trimester abortion or with 1 or more late pregnancy loss were considered for this study. In order to evaluate the causes of RPL we looked for chromosomal, endocrine, chronic inflammatory, and infectious alterations. 90 patients affected by unexplained RPL were enrolled and tested for hemostatic alterations. These women were tested for inherited and/or acquired thrombophilia by MTHFR C677T gene polymorphism, factor V Leiden gene polymorphism, PTHRA20210G gene polymorphism, protein S deficiency, protein $\mathrm{C}$ deficiency, antithrombin III deficiency, lupus anticoagulant, and anticardiolipin antibodies Ig G and Ig M.

Results: Acquired and/or inherited thrombophilia are strongly associated with RPL when other common causes of miscarriage were excluded. $78 \%$ of tested women showed hemostatic abnormalities. Several women with combined thrombophilic defects were also identified by our data.

Conclusion: After a thorough evaluation of other causes of miscarriage women affected by RPL should be tested for thrombophilia. Our data demonstrated $78 \%$ of women with one or combined thrombophilic conditions. Differences with previous studies should be related to difference in the inclusion and exclusion criteria and ethnic background. Because these patients often also show a hypercoagulable state, it an antithrombotic treatment before and during pregnancy may improve their clinical outcome (ie, secondary prevention of miscarriage and primary thromboprophylaxis).

Keywords: recurrent pregnancy loss, late pregnancy loss, thrombophilia, hypercoagulable state, hyperhomocysteinemia, factor V Leiden, prothrombin, antithrombotic drugs

\section{Background}

Recurrent fetal loss (RPL) is one of the most common causes of sterility. In a recent study we underlined the relevant role of d-dimer, as a marker of hypercoagulable state, in identifying thrombophilia in women affected by primary or secondary sterility (Di Micco et al 2004).

In 1999 Brenner et al (1999) identified thrombophilia as a principal cause in more than $40 \%$ of women affected by RPL. Further studies underlined a pathogenetic role of inherited thrombophilia in women affected by RFL. Sanson et al (1996) reported an increased frequency of antithrombin III, protein $\mathrm{C}$, and protein $\mathrm{S}$ deficiency in 
women with RPL, while several studies underlined the role of inherited thrombophilia (in particular related to factor $\mathrm{V}$ Leiden gene polymorphism and prothrombin A20210G gene polymorphism) in the pathophysiology of recurrent pregnacy loss (Brenner et al 1999; Carp et al 2002; Finan et al 2002; Grandone et al 1997; Greer 2003; Martinelli et al 2000; Pabinger et al 2000; Pickering et al 2001; Pihusch et al 2001; Rai et al 2001; Reznikoff-Etievan et al 2001; Younis et al 2001).

Yet increasing evidence is available on the relationship between hyperhomocysteinemia and methylenetetra-hydro-folate reductase (MTHFR) C677T gene polymorphism and unexplained recurrent pregnancy loss. Several reports have described an association between early recurrent pregnancy loss and hyperhomocysteinemia and/or MTHFR C677T gene polymorphism (Fatini et al 2000; Lissak et al 1999; Nelen et al 1997; Wouters et al 1993).

Acquired thrombophilia has also been associated with RPL. Dossenbach-Glaninger et al (2004) revealed that elevated maternal plasma levels of clotting factor VIII tend to be associated with an increased risk of RPL. Several studies have shown an association between RPL and primary or secondary antiphospholipid syndrome (Asherson and Cervera 1994; Chakrabarti et al 1999; Das et al 1991; Higashino et al 1998; Infante-Rivard et al 1991; Noble et al 2005). On this topic, a rare condition, acquired deficiency of clotting factor XII, has been described which Braulke et al (1993) identified for the first time a factor XII deficiency in RPL. Subsequently, Jones et al (2001) reported acquired factor XII deficiency in a subpopulation of women with antiphospholipid antibodies and RPL. Acquired clotting inhibitors, such as antibodies directed toward clotting factors, may induce an acquired clotting factors deficiency. In this field, we recently identified an unusual clinical presentation of antiphospholipid syndrome with clotting factor XII deficiency and the presence of antibodies to factor XII in a young woman with unexplained recurrent RPL (D’Uva et al 2005).

However, reported studies often do not evaluate other causes of miscarriages in their inclusion and exclusion criteria. So the aim of our study was to investigate the role of inherited thrombophilia in patients with RPL after exclusion of other causes of RPL.

\section{Patients and methods}

\section{Patients}

\section{Patient selection}

Starting from 1 January 2005, we selected 250 outpatients women referred to our Sterility Center for infertility owing to RPL. All patients with 2 or more first trimester abortions or with 1 or more late pregnancy loss were considered for this study. In order to evaluate the causes of RPL we looked for chromosomal alterations, endocrine diseases, chronic inflammatory, and infectious diseases.

All patients underwent karyotype study in order to detect several chromosomal aberrations such as balanced translocations. The uterine cavity was evaluated anatomically by transvaginal ultrasound scan and hysterosalpingography and/or hysteroscopy in order to detect mullerian malformations or the presence of fibroids or polyps. Endocrinological assessment included screening for diabetes, hypothyroidism, hypopituitarism, hyperprolactinemia, luteal insufficiency, and polycystic ovarian syndrome (PCOS). Basal FSH, LH, and estradiol, and luteal phase progesterone, TSH, prolactin levels, and fasting glucose were evaluated in all patients. In addition, trans-vaginal USG and androgens levels were assessed to look for PCOS. Chronic inflammation owing to immunological disease such as erythematosus lupus, rheumatoid arthritis, and systemic sclerosis were also studied. Serum level of antinuclear antibodies (ANA), antimithocondrial antibodies (AMA), and smooth muscle cells antibodies (SMA), rheumatoid arthritis, and levels of $\mathrm{C}$ reactive protein were assessed. Infective disease owing to Chlamydia spp, was evaluated by specific Chlamydia assays, associated with serological levels of specific IgG and IgM against Chlamydia spp. Moreover patients affected by obesity were excluded, in particular women with body mass index $>27$ were not enrolled in the study. Table 1 summarizes exclusion criteria.

\section{Patient group}

After this screening we selected 115 patients affected by unexplained RPL. These women were tested for inherited and/or acquired thrombophilia by methylene-tetra-hydrofolate

Table I Inclusion and exclusion criteria of selected subjects

\begin{tabular}{ll}
\hline Inclusion criteria & Exclusion criteria \\
\hline 2 or more first trimester abortion & Hypopituitarism \\
I or more late pregnancy loss & Hypothyroidism \\
Normal karyotype & Hyperprolactinemia \\
Normal uterine cavity & Luteal insufficiency \\
Tubal patency & PCOS \\
& Inflammatory or infectious disease \\
& Diabetes \\
& Obesity \\
& Single abortion \\
\hline
\end{tabular}

Abbreviation: PCOS, polycystic ovarian syndrome. 
reductase (MTHFR) C677T gene polymorphism, factor V Leiden gene polymorphism, PTHRA20210G gene polymorphism, protein $\mathrm{S}$ deficiency, protein $\mathrm{C}$ deficiency, antithrombin III deficiency, lupus anticoagulant, and anticardiolipin antibodies Ig $\mathrm{G}$ and Ig M.

\section{Methods}

Whole blood samples were collected from all selected subjects in the study by venipuncture from antecubital vein in order to screen for possible involvement hemostasis alteration.

\section{First blood sample}

A whole blood sample $(5 \mathrm{~mL})$ was collected in EDTA by venipuncture of antecubital vein. DNA was extracted using the "NUCLEON BACC" kit (Amershan, Germany). Patients were screened for the following mutations: Factor V G1691A gene polymorphism, A20210G gene polymorphism of the prothrombin, and C677T gene polymorphism of MTHFR using PCR amplification with specific primers and the Light Cycler apparatus (Roche, Italy).

\section{Second blood sample}

A second blood sample $(5 \mathrm{~mL})$ was collected in a Vacutainer tube containing $0.129 \mathrm{M}$ sodium citrate; the ratio of anticoagulant to blood was $1 / 9 \mathrm{v} / \mathrm{v}$.

Plasma activity of antithrombin III and protein S were evaluated with commercial kits (Bhoeringer, Germany) as was plasma protein S activity (Biopool, Sweden).

Anticardiolipin antibodies Ig G and IgM were detected by an ELISA method (anticariolipine Bouty, Italy).

Lupus anticoagulant assays were performed using a DRVVT kit (Screen and Confirm, Gradipore, North Ryde, Australia) and according to the recommendations of the International Society of Thrombosis and Haemostasis.

\section{Control group}

Seventy-five healthy age-matched subjects were enrolled as a control group. We included patients with one or more successful pregnancy and without gestational complication (intrauterine growth restriction, stillbirth, and abruptio placentae) or any abortion. We excluded patients with previous arterial and/or venous thrombosis. We also excluded subjects with first degree parents affected by arterial and/or venous thrombosis before than 65 years old.

\section{Statistical analysis}

Statistical analysis was based on Pearson chi-square (Npar tests); differences were considered to be significant if $\mathrm{p}<0.05$.

\section{Results}

The characteristics of study and control groups are summarised in Table 2. Patients were well matched according to age. Ninety patients showed alteration of hemostasis with a trend toward thrombophilia. Eighty-three patients with 2 or more early pregnancy losses and 7 patients with 1 or more late pregnancy loss were enrolled. Patients with RPL showed more gestations (median 3.1 vs 1.7 of control group), although only $10 \%$ ended with delivery of a living neonate.

Thrombophilia was present in $90 / 115$ patients $(78 \%)$ of study group compared with 12/75 (16\%) of control subjects ( $\mathrm{p}<0.001$, significant, s). Eighty-four patients showed isolated hemostatic abnormalities with a trend toward hypercoagulable state, while 6 patents showed combined defects inducing a hypercoagulable state, too.

The evaluation of the analyzed thrombophilic conditions revealed that factor $\mathrm{V}$ Leiden heterozygosity was present in 6/115 (5.2\%) patients with RPL vs $1 / 75$ (1.3\%) subjects of control group (p 0.091, ns); factor V Leiden homozygosity was absent in both study and control groups

Table 2 Frequency of thrombophilic alterations in women with RPL without other causes of miscarriage and control group

\begin{tabular}{llll}
\hline & Study group (I I 5 patients) & Control group (75 patients) & P \\
\hline MTHFR C677T homozigosity & $35 / 115(30.0 \%)$ & $7 / 75(9.3 \%)$ & $<0.001, \mathrm{~s}$ \\
FVL heterozigosity & $6 / 115(5.2 \%)$ & $1 / 75(1.3 \%)$ & $0.09, \mathrm{~ns}$ \\
PTHRA202I0G heterozigosity & $18 / 115(15 \%)$ & $2 / 75(2.6 \%)$ & $0.001, \mathrm{~s}$ \\
PS deficiency & $15 / 115(13 \%)$ & $2 / 75(2.6 \%)$ & $0.003, \mathrm{~s}$ \\
PC deficiency & $2 / 115(1.7 \%)$ & $0 / 75(0 \%)$ & $0.194, \mathrm{~ns}$ \\
APS & $10 / 115(8.6 \%)$ & $0 / 75(0 \%)$ & $0.003, \mathrm{~s}$ \\
Combined defects & $6 / 115(5.2 \%)$ & $0 / 75(0 \%)$ & $0.003, \mathrm{~s}$ \\
\hline
\end{tabular}

Abbreviations: RPL, recurrent pregnangy loss; MTHFR C677T, methylene tetrahydro folate reductase gene polymorphism; FVL, factorV Leiden gene polymorphism; PTHR A20210G, prothrombin gene polymorphism; PS, protein S deficiency; PC, protein C deficiency;APS, antiphospholipid syndrome. 
(statistical analysis not performed) (data not shown in Table 2). Prothrombin A20210G heterozygosity was present in $18 / 115(15 \%)$ patients with RPL vs $2 / 75$ (2.6\%) control (p 0.001, s), while prothrombin A20210G homozigosity was absent both in study and control groups (statistical analysis not performed) (data not shown in Table 2). MTHFR C677T homozygosity was present in $35 / 115(30.0 \%)$ patients with RPL vs 7/75 (9.3\%) control (p: 0.001, s).

We did not find any subjects with antithrombin III deficiency in either group (statistical analysis not performed) (data not shown in Table 2). Yet 2/115 (1.7\%) patients with RPL showed protein $\mathrm{C}$ deficiency vs no control subjects (p 0.194, ns). Protein S deficiency was present in 15/115 (13.0\%) of patients with RPL vs 2/75 (2.6\%) subjects of control subjects (p 0.003, s).

We also analyzed combined thrombophilic defects in our population. Of women affected by RPL, 6/115 (5.2\%) showed combined thrombophilic defects compared with no control subjects ( $\mathrm{p} 0.023, \mathrm{~s}$ ). Analysis of combined thrombophilia underlined these combined defects: 2 subjects showed protein S deficiency associated with MTHFR C677T homozygosity, 2 subjects showed protein $\mathrm{S}$ deficiency associated with prothrombin A20210G heterozygosity, 1 subject showed MTHFR C677T homozygosity associated with prothrombin A20210G heterozygosity, and 1 subject showed factor V Leiden heterozigosity associated with prothrombin A20210G heterozygosity.

Acquired thrombophilia owing to antiphospolipid syndrome was detected in 10/115 (8.6\%) study group women compared with no control subjects ( $\mathrm{p} 0.003, \mathrm{~s})$. LAC was present alone in 4/115 (3.4\%) study group subjects; anticardiolipin antibodies (ie, IgG and/or IgM) were present alone in $4 / 115(3.4 \%)$ study group subjects. LAC and anticardiolipin antibodies were both present in $2 / 115$ study group subjects $(1.7 \%)$.

\section{Discussion}

Alteration of hemostasis with a trend toward thrombophilia has been frequently associated to RPL. Several published studies seem to differ not only on the frequency of specific alteration but also on the inclusion and exclusion criteria of enrolled patients. For this reason we may observe also great differences about the involvement of thrombophilia during the evaluation of patients with RPL (Robertson et al 2006). However, the incidence of thrombophilia seems to increase if any causes of miscarriage, such as endocrine alterations and uterine malformations, are also excluded from the study population (Sarig et al 2002). Our data confirmed this trend and showed a strong association, reaching nearly $78 \%$, of one or more thrombophilic defects in women with RPL if other common causes of miscarriage are excluded. Before evaluating thrombophilia in our selected patients, we excluded several conditions associated with miscarriage such as uterine malformation, anovulation, tube patency, endocrine diseases (ie, diabetes, hypothyroidism, hypopituitarism, hyperprolactinemia, luteal insufficiency, and PCOS), chronic and/or infectious diseases, and karyotype alterations.

Our data are also of interest if the frequency of specific thrombophilic defects is analyzed. We did not find a strong association with factor $\mathrm{V}$ Leiden gene polymorphism and RPL, while we did find an increased frequency of prothrombin A20210G gene polymorphism compared with control subjects and also with other studies. Our data for prothrombin A20210G gene polymorphism was statistically significant. However, these differences from previous studies may be linked also to ethnic background, according to published data on geographical distribution of these gene variants (Ioannou et al 2000; Rosendaal et al 1998; Sarig et al 2002; Zalavras et al 2003). This reason may also explain the strong impact of the homozygosity of the MTHFR C677T gene polymorphism, which is present in the homozygosity gene variants in nearly $30 \%$ of the enrolled patients. Although MTFHR C677T gene polymorphism is a well recognized thrombotic risk factor, in particular for venous thromboembolism, data on its role in the pathophysiology of RPL per se is still a matter of discussion because of the role of folic acid in homocysteine metabolism. From another point of view, the relevant incidence of the MTHFR C677T gene variant may also be due to ethnic background, and for this reason we may also observe an increased incidence of the TT genotype in the control group (ie, nearly 16\%) (Rozen 2001). However, from a metabolic point of view previous studies may underestimate the involvement of homocysteine metabolism and MTHFR C677T gene variants in RPL: usually MTHFR C677T gene variants are analyzed only if a thrombotic disease, such as RPL, is associated with hyperhomocysteinemia. Yet the role of folic acid fortification during pregnancy is well known (Andersson et al 1992; Bonnette et al 1998) for preventing neural tube defects (Smithells et al 1980), and this folic acid fortification may determine also a transient reduction in homocysteine levels during pregnancy.

We did not find in our population homozygosity for prothrombin A20210G or factor V Leiden gene polymorphisms, or antithrombin deficiency. However, we did find a low incidence of protein $\mathrm{C}$ deficiency in our population, 
and these data seem to be in agreement with the results of Sarig et al (2002).

An increased frequency (statistically significant) of protein $\mathrm{S}$ deficiency was also found in our population, highlighting again its relevance in these patients affected by RPL. These data are also in agreement with those previously reported (Sanson et al 1996; Sarig et al 2002).

We found also a statistically significant increased incidence of antiphospholipid syndrome in women with RPL compared with control, which agrees with published data (Sarig et al 2002).

Particular attention should also be paid to the incidence of combined defects in women with RPL, which reached nearly $10 \%$ of investigated women. This observation could be very interesting for future studies because previous case control studies have underlined a potential role of combined thrombophilic defects in women with late pregnancy loss (Gris et al 1999; Sotiriadis et al 2007). We observed that more than $60 \%$ of our patients showed not only late pregnancy loss, as already reported in the literature, but also early RPL. Because our data on the role of combined thrombophilic defects in women with RPL reach statistical significance, even though the reduced number of selected patients was few, the role of combined thrombophilic defects in women with any type of recurrent pregnancy should be analyzed in further studies.

Our data emphasizes the role of thrombophilia in women with RPL. Specific research on the alteration of hemostasis in this clinical setting is particularly relevant in order to establish a possible protocol that supports clinical surveillance and follow up associated with thromboprophylaxis of these patients, both for repeated pregnancy loss and deep venous thrombosis, which is still a common complication in pregnant women. For the frequency of thrombophilic defects, we confirmed a major role for protein S deficiency, antiphospholipid syndrome, prothrombin A20210G gene variants, and MTHFR C677T gene variants, which has been reported previously. Interestingly, our data differ from those previously published in terms a reduced frequency of factor V Leiden gene variant. However, this gene variant should be also considered in this clinical setting because it has been observed in several of our patients, although its frequency did not reach statistical significance, probably because of a different ethnic distribution in our geographical area.

Alteration of hemostasis with a trend toward hypercoagulable state should be evaluated soon if patients affected by RPL do not show other causes of miscarriage. Because such patients may show an asymptomatic hyperocagulable state (Di Micco et al 2004) and because antithrombotic treatment in thrombophilic pregnant women with previous RPL has been shown to improve prognosis of related pregnancy (Brenner et al 2005a, b; Stephenson et al 2004), the possibility of starting early antithrombotic treatment (ie, before the beginning of pregnancy) should be studied, based on aspirin and/or low-molecular-weight heparin in order to evaluate further improvements in pregnancy outcome of thrombophilic women.

\section{Disclosures}

The authors have no conflicts of interest to declare.

\section{References}

Andersson A, Hultberg B, Brattstrom L, et al. 1992. Decreased serum homocysteine in pregnancy. Eur J Clin Chem Biochem, 30:377-9.

Asherson RA, Cervera R. 1994. "Primary", "secondary" and other variants of antiphospholipid syndrome. Lupus, 3:293-98.

Bonnette RE, Caudii MA, Boddie AM, et al. 1998. Plasma homocyst(e)ine concentrantions in pregnant and non pregnant women with controlled folate intake. Obstet Gynecol, 92:167-70.

Braulke I, Priggmayer M, Melloh P, Hinney B, Kostering H, Gunther H. 1993. Factor XII (Hageman) deficiency in women with abitual abortion: new subpopulation of recurrent aborters? Fertil Steril, 53:98-101.

Brenner B, Hoffan R, Carp H, et al; for the LIVE-ENOX Investigators. 2005b. Efficacy and safety of two doses of enoxaparin in women with thrombophilia and recurrent pregnancy loss: the LIVE-ENOX Study. $J$ Throm Haemost, 3:1-2.

Brenner B, Hoffman R, Carp H, et al. 2005a. Efficacy and safety of two doses of enoxaparin in women with thrombophilia and recurrent pregnancy loss: the LIVE-ENOX study. J Thromb Haemost, 3:227-9.

Brenner B, Sarig G, Weiner Z, et al. 1999. Thrombophilic polymorphisms are common in women with fetal loss without apparent cause. Thromb Haemost, 82:6-9.

Carp H, Salomon O, Seidman D, et al. 2002. Prevalence of genetic markers for thrombophilia in recurrent pregnancy loss. Hum Reprod, 17:1633-7.

Chakrabarti S, Bhunia C, Bhattacharya DK. 1999. The prevalence of antiphospholipid antibodies in cases of recurrent pregnancy loss. JAPI, 47:496-8.

D’Uva M, Strina, I, Mollo A, et al. 2005. Acquired factor XII deficiency in a woman with recurrent pregnancy loss: working on a differential diagnosis in a single case. J Transl Med, 3:43.

Das I, Vasishta K, Dash S. 1991. Study of lupus anticoagulant in pregnant women with recurrent abortion. Aust N Z J Obstet Gynecol, 31:323-6.

Di Micco P, D’Uva M, Strina I, et al. 2004. The role of d-dimer as first marker of thrombophilia in women affected by sterility: implications in pathophysiology and diagnosis of thrombophilia-induced sterility. J Transl Med, 2:38.

Dossenbach-Glaninger A, von Trotsenburg M, Krugluger W, et al. 2004. Elevated coagulation factor VIII and the risk for maternal early pregnancy loss. Thromb Haemost, 91:694-9.

Fatini C, Gensini F, Battaglini B, Prisco D, Cellai AP, Fedi S. 2000. Angiotensin convertine enzyme DD genotype, angiotensin - receptor CC genotype, and hyperhomocysteinemia increase first-trimester fetal-loss susceptibility. Blood Coagul Fibrinolysis, 11:657-62.

Finan RR, Tamim H, Ameen G, et al. 2002. Prevalence of factor V G1691A (factor V-Leiden) and prothrombin G20210A gene mutations in a recurrent miscarriage population. Am J Hematol, 71:300-5. 
Grandone E, Margaglione M, Colaizzo D, et al. 1997. Factor V Leiden is associated with repeated and recurrent unexplained fetal losses. Thromb Haemost, 77:822-4.

Greer IA. 2003. Thrombophilia: implications for pregnancy outcome. Thromb Res, 2152:1-9.

Gris JC, Quere I, Monpeyroux F, et al. 1999. Case control study of the frequency of thrombophilic disorders in couple with late pregnancy loss and no thrombotic antecedent. The Nimes Obstetricians and Haematologists Study (NOHA ${ }^{5}$ ). Thromb Haemost, 81:891-9.

Higashino M, Takakuwa K, Arakawa M, et al. 1998. Anticardiolipin antibody and anti-cardiolipin beta-2-glycoprotein I antibody in patients with recurrent fetal miscarriage. J Perinat Med, 26:384-9.

Infante-Rivard C, David M, Gauthier R, et al. 1991. Lupus anticoagulants, anticardiolipin antibodies, and fetal loss. A case-control study. NEng J Med, 325:1063-9.

Ioannou HV, Mitsis M, Eleftjeriou A, et al. 2000. The prevalence of factor $\mathrm{V}$ Leiden as a risk factor for venous thromboembolism in the population of North-Western Greece. Int Angiol, 19:314-18.

Jones DW, Mackie IJ, Gallimore MJ, et al. 2001. Antibodies to factor XII and recurrent fetal loss in patients with the anti-antiphospholid syndrome. Br J Haematol, 113:550-2.

Lissak A, Sharon A, Fruchter O, et al. 1999. Polymorphism for mutation of cytosine to thymine at location 677 in the methylenetetrahydrofolate reductase gene is associated with recurrent early fetal loss. Am J Obstet Gynecol, 181:126-30.

Martinelli I, Taioli E, Cetin I, et al. 2000. Mutations in coagulation factors in women with unexplained late fetal loss. N Engl J Med, 343:1015-8.

Nelen WLDM, van der Molen EF, Blom HJ, et al. 1997. Recurrent early pregnancy loss and genetic-related distrubances in folate and homocysteine metabolism. Br J Hosp Med, 58:511-3.

Noble LS, Kutteh WH, Lashey N, et al. 2005. Antiphospholipid antibodies associated with recurrent pregnancy loss: prospective, multicenter, controlled pilot study comparing treatment with low molecular weight heparin versus unfractioned heparin. Fertil Steril, 83:684-90.

Pabinger I, Nemes L, Rintelen C, et al. 2000. Pregnancy associated risk for venous thromboembolism and pregnancy outcome in women homozygous for factor V Leiden. Hematol J, 1:37-41.

Pickering W, Marriott K, Regan L. 2001. G20210A prothrombin gene mutation: prevalence in a recurrent miscarriage population. Clin Appl Thromb Haemost, 7:25-8.

Pihusch R, Buchholz T, Lohse P, et al. 2001. Thrombophilic gene mutations and recurrent spontaneous abortion: prothrombin mutation increases the risk in the first trimester. Am J Reprod Immunol, 46:124-31.
Rai R, Shlebak A, Cohen H, et al. 2001. Factor V Leiden and acquired activated protein $\mathrm{C}$ resistance among 1000 women with recurrent miscarriage. Hum Reprod, 16:961-5.

Reznikoff-Etievan MF, Cayol, V, Carbonne B, et al. 2001. Factor V Leiden and G20210A prothrombin mutations are risk factors for very early recurrent miscarriage. BJOG, 108:1251-4.

Robertson L, Wu O, Langhorne P, et al. 2006. The Thrombosis: risk and assessment of thrombophilia screening (TREATS) study. Thrombophilia in pregnancy: a systematic review. $\mathrm{Br} J$ Haematol, 132:171-96.

Rosendaal FR, Doggen CJ, Zivelin A, et al. 1998. Geographic distribution of the $20210 \mathrm{G}$ to A prothrombin variant. Thromb Haemost, 79:706-8.

Rozen R. 2001. Polymorphisms of folate and cobalamine metabolism. In Homocysteine in Health and Disease Edited by Ralph Carmel and Donal W Jacobsen. Cambridge: Cambridge University Press. p. 259-69.

Sanson BJ, Fierich PW, Simioni P, et al. 1996. The risk of abortion and stillbirth in antithrombin-, protein $\mathrm{C}$, and protein $\mathrm{S}$ deficient women. Thromb Haemost, 75:387-8.

Sarig G, Younis JS, Hoffman R, et al. 2002. Thrombophilia is common in women with idiopathic pregnancy loss and is associated with late pregnancy wastage. Fertil Steril, 77:342-7.

Smithells RW, Sheppard S, Schorah CJ, et al. 1980. Possible prevention of neural tube defects by periconceptional vitamin supplementation. Lancet, 8164:339-40.

Sotiriadis A, Vartholomatos G, Pavlou M, et al. 2007. Combined thrombophilic mutations in women with unexplained recurrent miscarriage. Am J Reprod Immunol, 57:133-41.

Stephenson MD, Ballem PJ, Tsang P, et al. 2004. Treatment of antiphospholipid syndrome (APS) in pregnancy: a randomised pilot trial comparing low molecular weight heparin to unfractioned heparin. J Obstet Gynaecol Can, 26:729-34.

Wouters MG, Boers GH, Blom HJ, et al. 1993. Hyperhomocysteinemia: a risk factor in women with unexplained recurrent early pregnancy loss. Fertil Steril, 60:820-5.

Younis JS, Brenner B, Ohel G, et al. 2000. Activated protein C resistance and factor $\mathrm{V}$ Leiden mutation can be associated with first- as well as secondtrimester recurrent pregnancy loss. Am J Reprod Immunol, 43:31-5.

Zalavras ChG, Giotopoulou S, Dokou E, et al. 2003. Prevalence of the G20210A prothrombin gene mutation in Northwestern Greece and association with venous thromboembolism. Int Angiol, 22:55-7. 